\title{
Screen Time Effect on Insomnia, Depression, or Anxiety Symptoms and Physical Activity of School Students During COVID-19 Lockdown in Lebanon: A Cross Sectional Study
}

\author{
Bayan Tarek Abou Ali*, Nada Omar Saleh ${ }^{1 *}$, Hussein Walid Mreydem¹, Sajida Fawaz Hammoudi', \\ Taeyeop Lee, MD², Seockhoon Chung, MD, PhD², Souheil Hallit, PharmD, MSc, MPH, PhD ${ }^{3,4}$, \\ Pascale Salameh, PharmD, MPH, PhD, HDR ${ }^{4,5,6}$ \\ ${ }^{1}$ Faculty of Medical Sciences, Lebanese University, Beirut, Lebanon \\ 2Department of Psychiatry, Asan Medical Center, University of Ulsan College of Medicine, Seoul, Korea \\ ${ }^{3}$ Faculty of Medicine and Medical Sciences, Holy Spirit University of Kaslik (USEK), Jounieh, Lebanon \\ ${ }^{4}$ Institut National de Santé Publique, d'Epidémiologie Clinique et de Toxicologie-Liban (INSPECT-LB), Beirut, Lebanon \\ ${ }^{5}$ Department of Basic Sciences at the Faculty of Medical Sciences, Lebanese University, Beirut, Lebanon \\ ${ }^{6}$ Department of Primary Care and Population Health, University of Nicosia Medical School, Nicosia, Cyprus
}

Received: November 12, 2021

Revised: December 7, 2021

Accepted: December 10, 2021

Correspondence

Seockhoon Chung, MD, PhD

Department of Psychiatry, Asan Medical Center

University of Ulsan College of Medicine,

88 Olympic-ro 43-gil, Songpa-gu,

Seoul 05505, Korea

Tel +82-2-3010-3411

Fax +82-2-485-8381

E-mailsschung@amc.seoul.kr

Pascale Salameh, PharmD, MPH, PhD, HDR

Department of Primary Care

and Population Health,

University of Nicosia Medical School,

93 Agiou Nikolaou Street,

Engomi 2408 Nicosia, Cyprus

Tel +961-3-385542

E-mail pascalesalameh1@hotmail.com

*These authors contributed equally to this work.

ORCID

Bayan Tarek Abou Ali

https://orcid.org/0000-0002-8920-2957

Nada Omar Saleh

https://orcid.org/0000-0002-2795-3448

Hussein Walid Mreydem

https://orcid.org/0000-0002-0963-5139

Saiida Fawaz Hammoudi

https://orcid.org/0000-0002-6315-9209

Taeyeop Lee

https://orcid.org/0000-0002-0350-084X

Seockhoon Chung

https://orcid.org/0000-0002-9798-3642

Souheil Hallit

https://orcid.org/0000-0001-6918-5689

Pascale Salameh

https://orcid.org/0000-0002-4780-0772

@ This is an Open Access article distributed under the terms of the Creative Commons Attribution Non-Commercial License (https://creativecommons.org/licenses/by-nc/4.0) which permits unrestricted non-commercial use, distribution, and reproduction in any medium, provided the original work is properly cited.
Background and Objective Our aim is to investigate the impact of COVID-19 on screen time among Lebanese high school students (grades 9-12).

Methods An anonymous online questionnaire was distributed among 510 school students from different governorates in Lebanon; this included questions regarding screen time, food habits, and physical activity. Psychological symptoms were assessed using the Generalized Anxiety Disorder-7 items and Patient Health Questionnaire-9 items. Effects of screen time on sleep was evaluated using the Insomnia Severity Index and Bedtime Procrastination Scale.

Results Female students reported higher depression $(p=0.018)$ and anxiety $(p=0.023)$ than male students; however, there was no difference in their sleep. Insomnia, depression, and anxiety were highest among phone users. A screen time of more than 7 hours per day was significantly associated with higher depression (67.9\%), anxiety (61.6\%), insomnia (82.1\%), and bedtime procrastination. It also indicated a shift toward a less healthy diet and light exercise.

Conclusions Seven hours of screen was found to develop depression and anxiety, exercising less, eating a less healthy diet, and experiencing disturbed sleep among school adolescents.

Sleep Med Res 2021;12(2):101-109

Keywords COVID-19; Screen time; Adolescent; Insomnia; Depression.

\section{INTRODUCTION}

'Screen time' refers to the time spent on any screen, including smart phones, tablets, televisions, video games, computers, or wearable technology. The digital landscape continues to expand with advancement in technology. All screen types have become a major part of contemporary life [1]. Thus, concerns about how an increase in screen time affects an individual's different health aspects have also risen [2]. The available data suggested that screen time is correlated with obesity, hypertension, type 2 diabetes, myopia, depression, sleep disorders, and many other non-communicable diseases.

In March 2020, the World Health Organization (WHO) declared the novel coronavirus a global pandemic [3], and many countries have implemented strict quarantine and social distancing measures in an attempt to control the spread of the virus [4]. This was also observed in Lebanon, where the first case of the virus was reported on February 21, 2020 and a lockdown 
has been imposed since March 18, 2020. These measures forced people to spend more time at home, mainly on their screens, working, studying, socializing, and entertaining; in particular, the switch from classroom to e-learning played a major role in the increase in screen time. Almost everything suddenly moved to a digital platform, to be performed via a screen; all outdoor activities became desk-based [4], which increased screen time and posed risks of screen-related health problems and poor physical activity.

Previous studies on adolescents found that users with a high screen time were more than twice as likely to have been diagnosed with depression and/or anxiety, treated by a mental health professional, or have taken medication for a psychological or behavioral issue, besides being at an increased risk of short sleep duration, long sleep onset latency, and increased sleep deficiency $[5,6]$. The importance of sleep, physical activity and mental health during adolescence is widely recognized, as this is a period of significant growth and development $[7,8]$. And also, eating healthy food and nutrition are critical for the development of children [9]. Depression or mental health have an impact on their eating habits [10], thus it is important to monitor food consumption and nutrition and treat this issue among children.

Several articles have reported the impact of COVID-19 pandemic on sleep, eating food, physical activity, depression, and anxiety; however, to our knowledge, no such studies have been conducted in Lebanon. Furthermore, most screen-related health problems have only been assessed in developed countries, and there is a lack of such information in underdeveloped countries such as Lebanon. Therefore, this study aimed to determine how an increased screen time can affect sleep, bedtime procrastination, depression, anxiety, eating habits, and physical activity among school students from grades 9-12.

\section{METHODS}

\section{Participants and Procedure}

An anonymous online survey was conducted. This study employed a descriptive analytical cross-sectional design. We collected responses from 510 Lebanese school students from February 7 to February 14, 2021; participation and responding to the survey were voluntary. The study protocol was approved by the Institutional Review Board of Hôpital Psychiatrique de la Croix (HPC-002-2021), and written informed consent was waived. Sociodemographic information including age, sex, weight, height, and region of residency were obtained.

\section{Assessment}

\section{Measuring screen time}

Direct questions were asked to school students in order to measure the amount of screen time they spent on different screen types and identify the type of device they used. Participants were also asked to specify their purpose behind spending most of their time on screens (e.g., studying, watching school lectures, social media, or entertainment).

\section{Measuring physical activity, body mass index, and food}

By asking "How do you describe your average physical activity?", participants were asked to specify their degree of physical activity as "light" (e.g., walking slowly [i.e., shopping, walking around the office], sitting at your computer, making the bed, eating, preparing food, or washing dishes), "moderate" (e.g., sweeping the floor, walking briskly, slow dancing, vacuuming, washing windows, or shooting a basketball), or "vigorous" (e.g., running $>5$ miles per hour, swimming, shoveling, soccer, jumping rope, carrying heavy loads [e.g., bricks]). We also determined students' food consumption by asking "During this month, your food mainly consisted of..."; respondents' responses included "healthy food" (e.g., vegetables, fruits, beans, chicken, or fish), "sometimes healthy food," or "unhealthy food" (e.g., fast food, fries, or sweets). Data about weight and height were obtained to calculate participants' body mass index (BMI), which is a classification used as per WHO's guidelines [11] for children aged 5-19 years old.

\section{Depression and anxiety}

Patient Health Questionnaire-9 items (PHQ-9) was used to assess participants' depression status. It consisted of nine questions with scores ranging from zero (not at all) to three (almost every day). Total scores were calculated for each participant; the maximum score was 27 . Depression severity was categorized as follows: none (0-4), mild (5-9), moderate (10-14), moderately severe (15-19), and severe (20-27) [12]. In this study, we used the Arabic version of the PHQ-9 [13] and defined clinical depression as a PHQ-9 score of $\geq 10$. To assess anxiety, the Generalized Anxiety Disorder-7 items (GAD-7) questionnaire was used. It comprised seven items, and scores range from zero (never) to three (nearly every day); the maximum score was 21 . Scores of 5, 10, and 15 were considered cut-off points for mild, moderate, and severe anxiety, respectively [14]. In this study, we used the Arabic version of the GAD-7 [13] and defined clinical anxiety as a GAD-7 score of $\geq 10$.

\section{Assessing sleep}

Insomnia and bedtime procrastination were evaluated in the sleep section of the questionnaire. For examining insomnia, the Insomnia Severity Index (ISI) was used; scores ranged from zero to four for each question, and the maximum score was 28. Participants' insomnia levels were classified as follows: clinically insignificant insomnia (0-7), subthreshold insomnia (8-14), and clinical insomnia (moderate severity $=15-21$; severe $=22-28$ ) [15]. In this study, we used the Arabic version of the ISI scale and defined clinical insomnia as an ISI score of $\geq 8$. 
To estimate bedtime procrastination, the Bedtime Procrastination Scale was used [16]. It consists of nine questions, with scores ranging from one (almost never) to five (almost always). Questions 2, 3, 7, and 9 were reverse scored (as indicated in the scale's manual). After summation of scores, participants with the highest score were considered as having the longest bedtime procrastination. The Arabic version was not formally published, and we translated this scale to Arabic with the permission of the original developers.

\section{Statistical Analysis}

The Mann-Whitney U-test and chi-square test for continuous variables and categorical variables were performed for between groups analyses, respectively. Spearman correlation analysis was

Table 1. Participants' demographic characteristics $(n=510)$

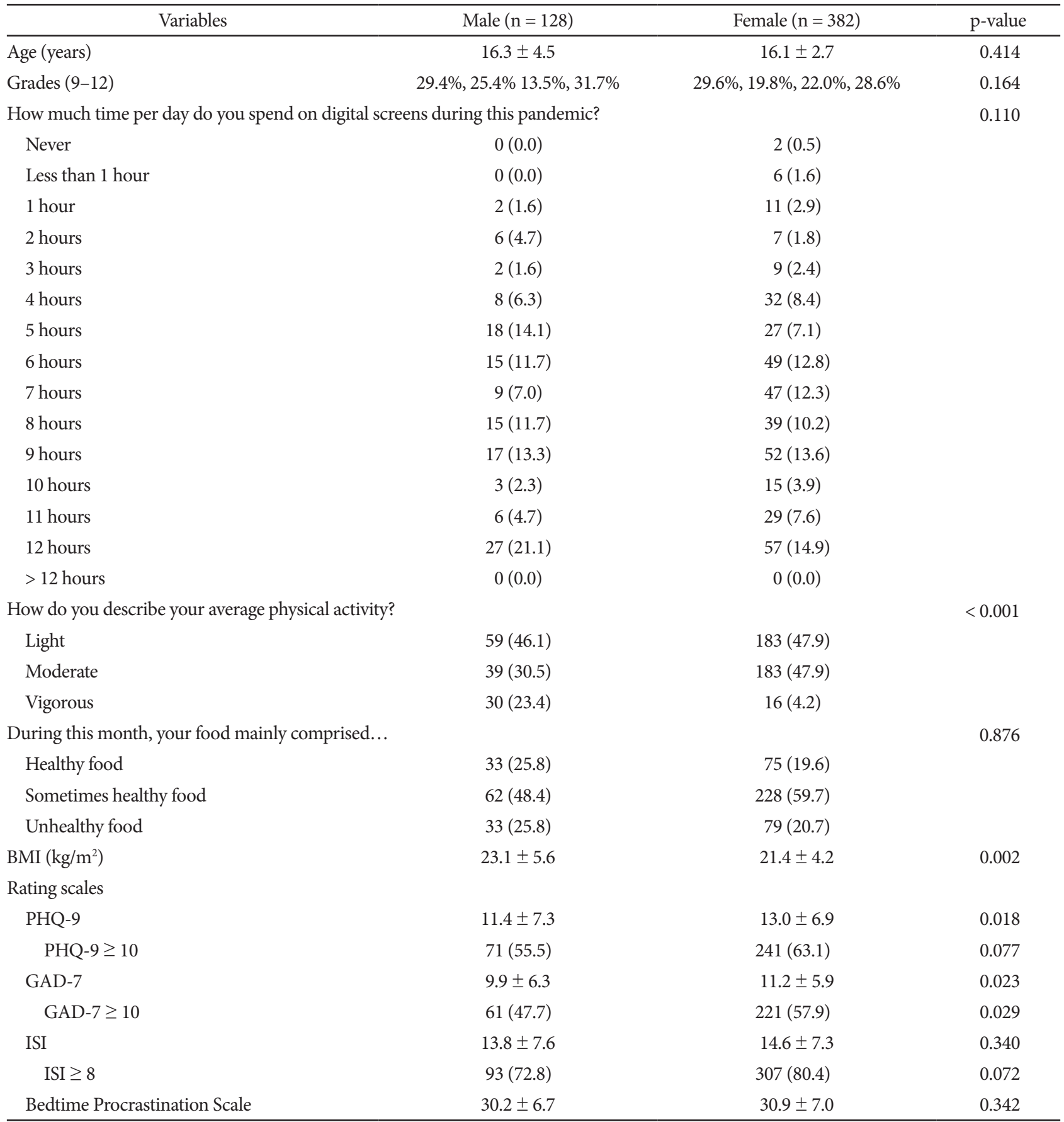

Data are presented as mean \pm standard deviation or $\mathrm{n}(\%)$.

BMI, body mass index; PHQ-9, Patient Health Questionnaire-9 items; GAD-7, Generalized Anxiety Disorder-7 items; ISI, Insomnia Severity Index. 
conducted to examine the relationship among continuous demographic variables and symptoms rating scales, since the distributions of PHQ-9 and GAD-7 scale scores were not within normal limits. To define the problematic screen time in accordance with the psychiatric symptoms, the Receiver Operating Characteristic (ROC) analysis was conducted. Stepwise logistic regression analysis was performed after adjusting for students' demographic variables to explore the determining factors of problematic screen time. Demographic characteristics and clinical data were summarized as mean \pm standard deviation. The level of significance for all the analyses was defined as two-tailed, $p<$ 0.05 . SPSS version 21.0 for Windows (IBM Corp., Armonk, NY, USA) was used for statistical analysis.

\section{RESULTS}

In total, 510 secondary level school students from grades 9-12 participated in this online survey. Among these, 382 (74.9\%) were females, and there was no significant difference in age between male and female students. Respondents were from Beirut $(\mathrm{n}=$ 41, 8.0\%), Mount Lebanon ( $\mathrm{n}=27,5.3 \%)$, North Lebanon $(\mathrm{n}=$ $77,15.1 \%)$, Akkar $(n=6,1.2 \%)$, South Lebanon $(n=71,13.9 \%)$, Nabatieh $(\mathrm{n}=3,0.6 \%)$, Bekaa $(\mathrm{n}=276,54.1 \%)$, and BaalbakHermel $(n=9,1.8 \%)$. Their reported screen time per day is pre- sented in Table 1.

Concerning participants' average physical activity, male students reported a higher proportion of vigorous activity compared to female students. There was no significant difference in food choice and BMI between male and female students. Female students reported a significantly higher level of depression (PHQ-9, $\mathrm{p}=0.018)$ and generalized anxiety (GAD-7, $\mathrm{p}=0.023)$ compared to male students. However, there was no significant difference in terms of insomnia (ISI, $\mathrm{p}=0.340$ ) and bedtime procrastination $(\mathrm{p}=0.342)$.

\section{Levels of Mental Health and Screen Time}

Fig. 1 shows the clinical levels of insomnia (ISI score $\geq 8$ ), generalized anxiety (GAD-7 score $\geq 10$ ), and depression (PHQ-9 score $\geq 10$ ) for total screen time per day. Overall, the clinical levels of insomnia, anxiety, and depression increased as the screen time increased.

We asked students to report their screen time for each electronic device, including phones, tablets/iPads, TVs/PlayStations/ Xbox, and/or computers. Fig. 2 shows the clinical levels of insomnia, anxiety, and depression for each screen time spent on electronic devices including phones (Fig. 2A), tablets/iPads (Fig. 2B), TVs/PlayStations/Xbox (Fig. 2C), and computers (Fig. 2D). Overall, an increase in the levels of psychiatric symptoms was observed only for phone screen time. Contrarily, for other elec-

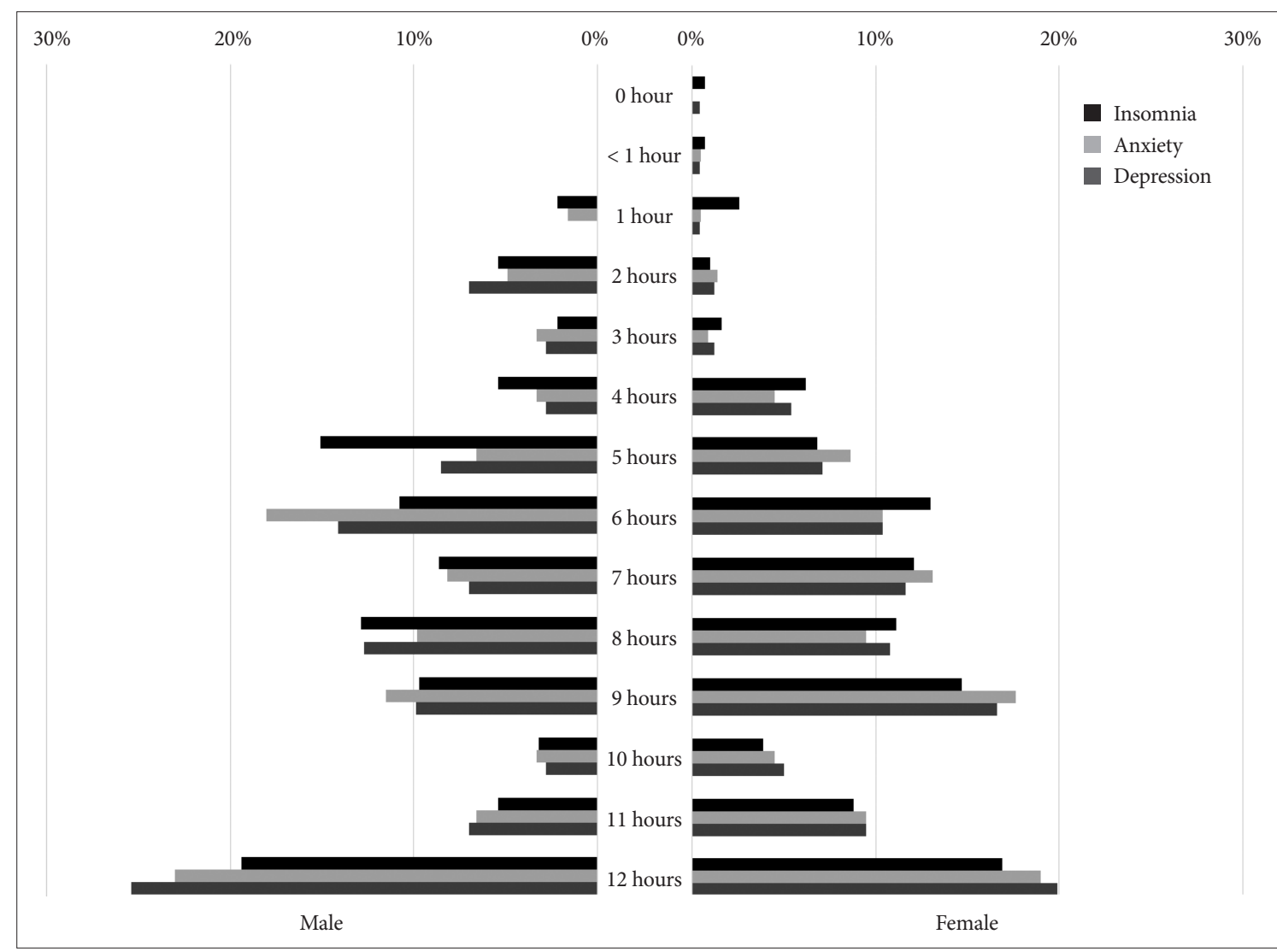

Fig. 1. Screen time and the clinical levels of clinical depression, anxiety, and insomnia. 


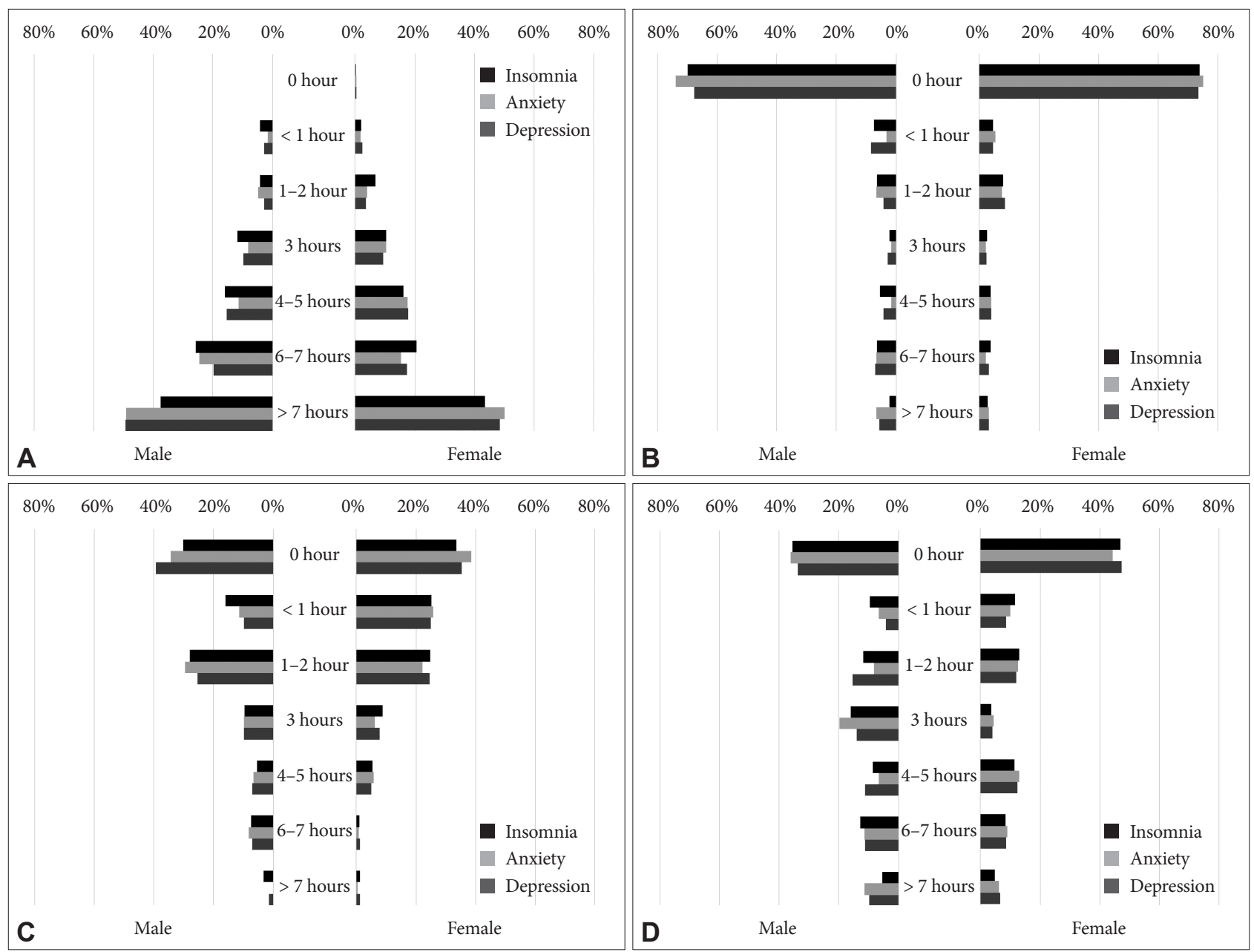

Fig. 2. Clinical levels of insomnia, anxiety, and depression for screen time of each electronic devices. A: Phone using time. B: Tablet/iPad using time. C: TV/PlayStation/Xbox using time. D: Computer using time.

tronic devices, the levels of psychiatric symptoms decreased as screen time increased.

\section{Exploring Problematic Screen Time}

We conducted a ROC analysis to determine the problematic screen time for mental health among school students. The ROC analysis revealed that seven hours of screen time was indicative of clinical levels of depression (area under curve $[\mathrm{AUC}]=0.672$, sensitivity $=0.83$, specificity $=0.38)$, anxiety $(A U C=0.637$, sensitivity $=0.83$, specificity $=0.36$ ), and insomnia $(A U C=0.606$, sensitivity $=0.78$, specificity $=0.38)$. In this study, we defined seven hours of screen time as the problematic screen time. Between students who reported $<$ seven hours of screen time and $\geq$ seven hours of screen time, there was a significant difference in physical activity $(p=0.028)$ and food choice $(p=0.047)$. The severity of insomnia, depression, anxiety, and bedtime procrastination were significantly higher among students who reported $\geq$ seven hours of screen time compared to students who reported $<$ seven hours of screen time (Table 2).

After adjusting for students' sex, age, BMI, food constitution, physical activity, PHQ-9 score, and bedtime procrastination, the stepwise logistic regression analysis revealed that ISI (adjusted odds ratio [aOR]: 1.04, 95\% confidence interval [CI]: 1.0011.074 ) and generalized anxiety (aOR: 1.06, 95\% CI: 1.010-1.102) were significant predictors of seven hours of screen time.

We analyzed the difference in levels of clinical depression, anxiety, insomnia, bedtime procrastination, and BMI between students who reported $<$ seven hours of screen time and $\geq$ seven hours of screen time, based on their purpose of using a screen (Table 3).

\section{DISCUSSION}

In this study, we observed that a screen time of more than seven hours per day was significantly associated with higher depression, anxiety, insomnia, and bedtime procrastination. In addition, their insomnia and generalized anxiety were significant predictors of seven hours of screen time. The purpose of this study was to see how much increasing digital screen time affected sleep, 
Table 2. Difference in clinical variables based on 7 hours of screen time $(n=510)$

\begin{tabular}{|c|c|c|c|}
\hline Variables & $\begin{array}{l}\text { Screen time } \\
<7 \text { hours } \\
(\mathrm{n}=130)\end{array}$ & $\begin{array}{l}\text { Screen time } \\
\geq 7 \text { hours } \\
(\mathrm{n}=380)\end{array}$ & p-value \\
\hline Age (years) & $16.0 \pm 3.2$ & $16.2 \pm 3.3$ & 0.113 \\
\hline Sex (female) & $94(72.3)$ & $288(75.8)$ & 0.249 \\
\hline \multicolumn{3}{|c|}{ How do you describe your average physical activity? } & 0.028 \\
\hline Light & $50(38.5)$ & $192(50.5)$ & \\
\hline Moderate & $63(48.5)$ & $159(41.8)$ & \\
\hline Vigorous & $17(13.1)$ & $29(7.6)$ & \\
\hline \multicolumn{3}{|c|}{ During this month, your food was mainly comprised... } & 0.047 \\
\hline Healthy food & $37(28.5)$ & $71(18.7)$ & \\
\hline Sometimes healthy food & $70(53.8)$ & $220(57.9)$ & \\
\hline Unhealthy food & $23(17.7)$ & $89(23.4)$ & \\
\hline BMI $\left(\mathrm{kg} / \mathrm{m}^{2}\right)$ & $21.8 \pm 4.6$ & $22.1 \pm 4.9$ & 0.512 \\
\hline \multicolumn{4}{|l|}{ Rating scales } \\
\hline PHQ-9 & $10.2 \pm 7.3$ & $13.4 \pm 6.8$ & $<0.001$ \\
\hline PHQ-9 $\geq 10$ & $54(41.5)$ & $258(67.9)$ & $<0.001$ \\
\hline GAD-7 & $8.8 \pm 5.9$ & $11.6 \pm 5.9$ & $<0.001$ \\
\hline GAD-7 $\geq 10$ & $48(36.9)$ & $234(61.6)$ & $<0.001$ \\
\hline ISI & $11.9 \pm 7.5$ & $15.2 \pm 7.2$ & $<0.001$ \\
\hline ISI $\geq 8$ & $88(67.7)$ & $312(82.1)$ & $<0.001$ \\
\hline $\begin{array}{l}\text { Bedtime Procrastination } \\
\text { Scale }\end{array}$ & $29.1 \pm 7.0$ & $31.3 \pm 6.8$ & 0.001 \\
\hline
\end{tabular}

Data are presented as mean \pm standard deviation or $\mathrm{n}(\%)$. BMI, body mass index; PHQ-9, Patient Health Questionnaire-9 items; GAD-7, Generalized Anxiety Disorder-7 items; ISI, Insomnia Severity Index.

bedtime procrastination, depression, and physical activity among school students during the pandemic. The findings are important because combating the pandemic requires not just social isolation and restricted measures, but also keeping a robust immune system to help guard against infection. Sleep, mental health, and physical activity all play important roles in the function of the immune system; thus, tracking these would be helpful in establishing a better defense against COVID-19 virus [17-19].

In our study, we found that levels of psychological symptoms increased only with an increase in screen time for phones. This is consistent with two previous studies performed with university students in Turkey, wherein a positive significant relationship between smartphone addiction and quality of sleep or depression was statistically determined [20,21]. However, other electronic devices had an inversely proportional relationship between screen time and levels of psychiatric symptoms.

Our results showed that females scored significantly higher for anxiety $(11.2 \pm 5.9)$ and depression $(13.0 \pm 6.9)$ than males $(9.9 \pm 6.3$ for anxiety; $11.4 \pm 7.3$ for depression) with $\mathrm{p}=0.023$ and $\mathrm{p}=0.018$, respectively. Studies have shown that females tend to be more reticent while males may shift their attention to other affairs as the screen time increases [22]. This might explain why females in our study were more depressed and anxious. Considering seven hours as the problematic screen time, anxiety and depression were higher in participants spending $\geq$ seven hours on screen as compared to those spending < seven hours, with a $\mathrm{p}<0.001$ for both GAD-7 and PHQ-9. Individuals with higher screen time had significantly elevated risks of depression; this was also reported in a meta-analysis of 12 cross-sectional and seven longitudinal studies [22]. In a 12-country study on screen usage, researchers discovered that every additional hour spent on a screen every day increases teenagers' psychiatric symptoms by 0.04 on a 5 -point scale [23].

Regarding sleep, ISI and bedtime procrastination showed no difference between males and females. When adjusting the results to a cut off of seven hours, ISI and bedtime procrastination were significant, with $p<0.001$ and $p=0.001$, respectively, between those using a screen for $<$ seven hours and those using one for $\geq$ seven hour. Longer use of screen at bedtime was linked to poor sleep quality and longer bedtime procrastination [24]. A study investigating screen time of middle-school aged children found that one hour or more of evening screen time causes a 3.4 times significant increase $(\mathrm{p}<0.01)$ in bedtime procrastination [25]. This may be contributed to time displacement, which means that the higher the time spent on screens, the lesser time available for an individual to sleep [26]. Other studies revealed that light stimulates alertness [27], and delays circadian rhythm [28]. Therefore, the increase in bedtime procrastination in students spending seven hours or more on screens may be attributed to time displacement theory or light alertness.

There was a significant difference in food choice $(p=0.047)$ and physical activity $(\mathrm{p}=0.028)$ when considering seven hours as the cut off; however, BMI showed no variation ( $\mathrm{p}=0.512)$. Our data showed that those using a screen for $\geq$ seven hours were performing more light exercises than those with a screen time of $<$ seven hours. A screen time of $\geq$ seven hours was linked with more depression (13.4 \pm 6.8 on PHQ-9). A systematic review and meta-analysis about associations between screen time-based sedentary behavior and depression disclosed that most participants with a sedentary lifestyle were more likely to have depression at a screen time threshold of $>2 \mathrm{~h} / \mathrm{d}$ [22], which is different from the seven-hour threshold that we determined as the problematic time. A follow-up study with children aged 11 years on screen use during school days and weekends (TV and computer) showed an increase in BMI after three years [29]. Our study was cross sectional by design, which may be the reason behind the insignificant BMI difference observed during the study period. A screen time of $\geq$ seven hours was associated with a decline in healthy food consumption, and an aggravation in eating "sometimes healthy food" and "unhealthy food." A systematic review reported a moderate association between screen time (particularly for TV) and a less healthy diet consist- 
Table 3. Difference in levels of clinical symptoms based on students' purpose of using a screen

\begin{tabular}{|c|c|c|c|}
\hline Variables & \multicolumn{2}{|c|}{ Values } & p-value \\
\hline \multicolumn{4}{|l|}{ For what purpose do you spend most of your time on screens? } \\
\hline A) Studying $(\mathrm{n}=284)$ & Screen time $<7$ hours $(\mathrm{n}=81)$ & Screen time $\geq 7$ hours $(n=203)$ & \\
\hline PHQ-9 $\geq 10$ & $37(45.7)$ & $141(69.5)$ & $<0.001$ \\
\hline GAD-7 $\geq 10$ & $29(35.8)$ & $135(66.5)$ & $<0.001$ \\
\hline ISI $\geq 8$ & $55(67.9)$ & $175(86.2)$ & $<0.001$ \\
\hline Bedtime Procrastination Scale & $28.9 \pm 6.9$ & $31.1 \pm 6.8$ & 0.013 \\
\hline BMI $\left(\mathrm{kg} / \mathrm{m}^{2}\right)$ & $21.9 \pm 5.6$ & $21.6 \pm 3.9$ & 0.687 \\
\hline B) Watching school lectures $(n=65)$ & Screen time $<7$ hours $(\mathrm{n}=10)$ & Screen time $\geq 7$ hours $(n=55)$ & \\
\hline PHQ-9 $\geq 10$ & $6(60.0)$ & $40(72.7)$ & 0.321 \\
\hline $\mathrm{GAD}-7 \geq 10$ & $7(70.0)$ & $37(67.3)$ & 0.590 \\
\hline ISI $\geq 8$ & $8(80.0)$ & $45(81.8)$ & 0.594 \\
\hline Bedtime Procrastination Scale & $31.4 \pm 8.1$ & $30.5 \pm 6.9$ & 0.716 \\
\hline $\mathrm{BMI}\left(\mathrm{kg} / \mathrm{m}^{2}\right)$ & $21.1 \pm 2.2$ & $21.1 \pm 3.4$ & 0.974 \\
\hline C) Social media $(n=67)$ & Screen time $<7$ hours $(\mathrm{n}=17)$ & Screen time $\geq 7$ hours $(n=50)$ & \\
\hline PHQ-9 $\geq 10$ & $6(35.3)$ & $33(66.0)$ & 0.027 \\
\hline GAD-7 $\geq 10$ & $7(42.2)$ & $30(60.0)$ & 0.143 \\
\hline ISI $\geq 8$ & $9(52.9)$ & $42(84.0)$ & 0.009 \\
\hline Bedtime Procrastination Scale & $28.7 \pm 7.8$ & $33.8 \pm 5.6$ & 0.005 \\
\hline $\operatorname{BMI}\left(\mathrm{kg} / \mathrm{m}^{2}\right)$ & $22.4 \pm 2.9$ & $21.7 \pm 6.3$ & 0.661 \\
\hline $\begin{array}{l}\text { D) Entertainment (video games, watching movies/TV } \\
\text { series, YouTube) }(\mathrm{n}=94)\end{array}$ & Screen time $<7$ hours $(\mathrm{n}=22)$ & Screen time $\geq 7$ hours $(n=72)$ & \\
\hline PHQ-9 $\geq 10$ & $5(22.7)$ & $44(61.1)$ & $<0.001$ \\
\hline GAD-7 $\geq 10$ & $5(22.7)$ & $32(44.4)$ & 0.055 \\
\hline ISI $\geq 8$ & $16(72.7)$ & $50(69.4)$ & 0.497 \\
\hline Bedtime Procrastination Scale & $29.3 \pm 6.7$ & $30.4 \pm 7.0$ & 0.499 \\
\hline $\operatorname{BMI}\left(\mathrm{kg} / \mathrm{m}^{2}\right)$ & $22.9 \pm 4.2$ & $22.7 \pm 5.7$ & 0.879 \\
\hline
\end{tabular}

Data are presented as mean \pm standard deviation or $\mathrm{n}(\%)$.

PHQ-9, Patient Health Questionnaire-9 items; GAD-7, Generalized Anxiety Disorder-7 items; ISI, Insomnia Severity Index; BMI, body mass index.

ing of a low intake of healthy food groups and higher energy intake [1]. Despite the fact that the Global Movement Behavioral Guidelines for children and adolescents recommended that young children aged 3-4 years should not engage in more than one hour of sedentary screen time, and that older children and adolescents aged 5-17 years should not engage in more than two hours of sedentary recreational screen time [30], this reality of spending half the day on the screen is inevitable as classes (which typically last for seven hours, even in a physical setting) have now moved online.

In this study, we defined seven hours of screen time as the problematic screen time using the ROC analysis based on psychiatric symptoms including insomnia, anxiety, and depression in school students. Our findings are in line with another study performed in the US in 2016 with a random sample of children and adolescents aged between 2-17 years, which reported that users with a higher screen time $(7+\mathrm{h} /$ day vs. $1 \mathrm{~h}$ /day) were twice as likely to develop depression, anxiety, or other behavioral and psychiatric issues. Additionally, an association between lower psychological well-being and screen time was found among users with a moderate screen time $(4 \mathrm{~h} /$ day). These findings were not observed in users with lower or zero hours of screen time [5].

In this study, the clinical levels of insomnia (male: $72.8 \%$; female: $80.4 \%$ ), depression (male: $55.5 \%$; female: $63.1 \%$ ), and anxiety (male: $47.7 \%$; female: $57.9 \%$ ) was very high among school students. It is important to note that these results were obtained during the second wave of the COVID-19 pandemic, when a second lockdown was imposed by the government following a huge increase in the number of infected individuals following the new year celebrations. This is also a period when Lebanon was facing an economic and political crisis. Additionally, six months ago, the Lebanese population witnessed a catastrophic event when an explosion occurred in Beirut on August 4, 2020, which killed more than 178 citizens; left more than 6500 injured and 300000 
homeless; and severely damaged critical health infrastructure and medical supplies. This might explain the high clinical levels of depression, anxiety, and insomnia among school students who have started questioning their academic future. We considered this a limitation of our study, along with the fact that no data were available regarding screen time in the pre-pandemic or pre-explosion period. Another limitation includes the actuality that the data were collected through an online survey, which might influence the objectivity of the results. Third, the Bedtime Procrastination Scale was not formally validated in Arabic. Finally, we asked participants whether they have own electronic device or not, since the device possession might be important factor. However, we cannot discriminate which electronic device was owned by participants, and we could not apply the responses in the statistical analysis. And also, it is not easy to discriminate the purpose of using a screen and it might lead the bias of the results.

In conclusion, we defined seven hours as the problematic screen time for school children, and observed that increased digital screen time impacted their sleep, bedtime procrastination, depression, and physical activity during the pandemic. We also found that psychiatric symptoms increased only when screen time for phones increased. These findings should be considered while defining the appropriate screen time and helping school students control their screen time, even during this time when online education is more prevalent.

\section{Availability of Data and Material}

The datasets generated or analyzed during the study are available from the corresponding author on reasonable request.

\section{Author Contributions}

Conceptualization: Hallit S, Salameh P. Data curation: Abou Ali BT, Saleh NO, Mreydem HW, Hammoudi SF. Formal analysis: Lee T, Chung S, Salameh P. Methodology: Lee T, Chung S, Salameh P. Project administration: Abou Ali BT, Mreydem HW. Software: Lee T, Chung S. Supervision: Chung S, Hallit S, Salameh P. Visualization: Abou Ali BT, Saleh NO, Mreydem HW, Hammoudi SF, Lee T. Writing-original draft: Abou Ali BT, Saleh NO, Mreydem HW, Hammoudi SF. Writing_review \& editing: Lee T, Chung S, Hallit S, Salameh P.

\section{Conflicts of Interest}

The authors have no potential conflicts of interest to disclose.

\section{Funding Statement}

None.

\section{REFERENCES}

1. Stiglic N, Viner RM. Effects of screentime on the health and well-being of children and adolescents: a systematic review of reviews. BMJ Open 2019;9:e023191.

2. Canadian Paediatric Society, Digital Health Task Force, Ottawa, Ontario. Screen time and young children: promoting health and development in a digital world. Paediatr Child Health 2017;22:461-77.

3. Pišot S, Milovanović I, Šimunič B, Gentile A, Bosnar K, Prot F, et al. Maintaining everyday life praxis in the time of COVID-19 pandemic measures (ELP-COVID-19 survey). Eur J Public Health 2020;30:1181-6.

4. Schmidt SCE, Anedda B, Burchartz A, Eichsteller A, Kolb S, Nigg C, et al. Physical activity and screen time of children and adolescents before and during the COVID-19 lockdown in Germany: a natural experiment. Sci Rep 2020;10:21780.

5. Twenge JM, Campbell WK. Associations between screen time and lower psychological well-being among children and adolescents: evidence from a population-based study. Prev Med Rep 2018;12:271-83.

6. Hysing M, Pallesen S, Stormark KM, Jakobsen R, Lundervold AJ, Sivertsen B. Sleep and use of electronic devices in adolescence: results from a large population-based study. BMJ Open 2015;5:e006748.

7. Xu F, Adams SK, Cohen SA, Earp JE, Greaney ML. Relationship between physical activity, screen time, and sleep quantity and quality in US adolescents aged 16-19. Int J Environ Res Public Health 2019;16: 1524.

8. O’Reilly M, Dogra N, Hughes J, Reilly P, George R, Whiteman N. Potential of social media in promoting mental health in adolescents. Health Promot Int 2019;34:981-91.

9. Proia P, Amato A, Drid P, Korovljev D, Vasto S, Baldassano S. The impact of diet and physical activity on bone health in children and adolescents. Front Endocrinol (Lausanne) 2021;12:704647.

10. Campisi SC, Krause KR, Chan BWC, Courtney DB, Bennett K, Korczak DJ, et al. Eating, sleeping and moving recommendations in clinical practice guidelines for paediatric depression: umbrella review. BJPsych Open 2021;7:e185.

11. WHO. BMI-for-age (5-19 years) [cited $2021 \mathrm{Dec} 22$ ]. Available from: https://www.who.int/tools/growth-reference-data-for-5to19-years/indicators/bmi-for-age.

12. Kroenke K, Spitzer RL, Williams JB. The PHQ-9: validity of a brief depression severity measure. J Gen Intern Med 2001;16:606-13.

13. Sawaya H, Atoui M, Hamadeh A, Zeinoun P, Nahas Z. Adaptation and initial validation of the patient health questionnaire-9 (PHQ-9) and the generalized anxiety disorder-7 questionnaire (GAD-7) in an Arabic speaking Lebanese psychiatric outpatient sample. Psychiatry Res 2016;239:245-52.

14. Spitzer RL, Kroenke K, Williams JB, Löwe B. A brief measure for assessing generalized anxiety disorder: the GAD-7. Arch Intern Med 2006;166:1092-7.

15. Morin CM, Belleville G, Bélanger L, Ivers H. The insomnia severity index: psychometric indicators to detect insomnia cases and evaluate treatment response. Sleep 2011;34:601-8.

16. Kroese FM, De Ridder DT, Evers C, Adriaanse MA. Bedtime procrastination: introducing a new area of procrastination. Front Psychol 2014; 5:611.

17. da Silveira MP, da Silva Fagundes KK, Bizuti MR, Starck É, Rossi RC, de Resende E Silva DT. Physical exercise as a tool to help the immune system against COVID-19: an integrative review of the current literature. Clin Exp Med 2021;21:15-28.

18. Besedovsky L, Lange T, Born J. Sleep and immune function. Pflugers Arch 2012;463:121-37.

19. Vasile C. Mental health and immunity (Review). Exp Ther Med 2020;20: 211.

20. Kaya F, Bostanci Daştan N, Durar E. Smart phone usage, sleep quality and depression in university students. Int J Soc Psychiatry 2021;67:40714.

21. Demirci K, Akgönül M, Akpinar A. Relationship of smartphone use severity with sleep quality, depression, and anxiety in university students. J Behav Addict 2015;4:85-92.

22. Wang X, Li Y, Fan H. The associations between screen time-based sedentary behavior and depression: a systematic review and meta-analysis. BMC Public Health 2019;19:1524.

23. Vandendriessche A, Ghekiere A, Van Cauwenberg J, De Clercq B, Dhondt K, DeSmet A, et al. Does sleep mediate the association between school pressure, physical activity, screen time, and psychological symptoms in early adolescents? A 12-country study. Int J Environ Res Public Health 2019;16:1072.

24. Christensen MA, Bettencourt L, Kaye L, Moturu ST, Nguyen KT, Ol- 
gin JE, et al. Direct measurements of smartphone screen-time: relationships with demographics and sleep. PLoS One 2016;11:e165331.

25. Kubiszewski V, Fontaine R, Rusch E, Hazouard E. Association between electronic media use and sleep habits: an eight-day follow-up study. Int J Adolesc Youth 2014;19:395-407.

26. Hale L, Guan S. Screen time and sleep among school-aged children and adolescents: a systematic literature review. Sleep Med Rev 2015; 21:50-8.

27. Cajochen C. Alerting effects of light. Sleep Med Rev 2007;11:453-64.

28. Khalsa SB, Jewett ME, Cajochen C, Czeisler CA. A phase response curve to single bright light pulses in human subjects. J Physiol 2003; 549:945-52.

29. Engberg E, Figueiredo RAO, Rounge TB, Weiderpass E, Viljakainen H. Heavy screen use on weekends in childhood predicts increased body mass index in adolescence: a three-year follow-up study. J Adolesc Health 2020;66:559-66

30. WHO. Guidelines on physical activity, sedentary behaviour and sleep for children under 5 years of age [cited 2021 Dec 7]. Available from: https://www.who.int/publications/i/item/9789241550536. 